\title{
Bronchoscopy Report: format and filing, images and exam correlation
}

\author{
L. Corbetta1, C. Mereu²
}

Monaldi Arch Chest Dis 2011; 75: 1, 14-18.

Keywords: Bronchoscopy, Report, Images, Computerized, Filing.

1 SOD di Broncologia diagnostico-interventistica, Azienda Ospedaliero-Universitaria Careggi, Firenze,

2 Struttura Complessa di Pneumologia dell'Azienda Ospedaliera, Ospedale Santa Corona di Pietra Ligure (SV), Italy.

Correspondence: Prof. Lorenzo Corbetta, Università degli Studi di Firenze, Direttore SOD di Broncologia diagnostico-interventistica, Azienda Ospedaliero-Universitaria Careggi, Viale Morgagni 85, 50134 Firenze, Italy; e-mail: lorenzo.corbetta@unifi.it

\section{Background}

In the endoscopy practice it is of the utmost importance that both medical reports and the details of the procedure performed are meticulously recorded for each and every patient. Pathological reports should be detailed whereas standard reports should be of a more general nature and should include the procedure carried out in order to obtain the sample as well as any other complications. points:

The final report must include the following

- Information of the medical team involved in the treatment of the patient.

- Information of any follow-up bronchoscopies.

- Documentation for quality control purposes.

- Planning of subsequent diagnostic interventions or treatments.

- Information of scientific interest.

The flexible bronchoscopy report is one of the tools permitting exchange of medical information in respiratory medicine and is an integral part of the medical record. Currently, there is no consensus on its content, and consequently, there are no recommendations. Recently a survey was carried out involving experts which showed that there are as many areas of divergence among physicians [1].

For more accurate information in the endoscopic field photographic images and video recordings are frequently consulted. New computerised technology has made the inclusion of multimedia information much easier and has significantly simplified the preparation, standardisation and electronic compilation of the bronchoscopic report. This document has the aim to harmonise the writing of bronchoscopy reports, to provide a tool consensual and complete, and valid from the medico-legal viewpoint.

\section{Computerised bronchoscopic records}

A computerised database is the ideal instrument for the recording and the subsequent retrieval of bronchoscopic information with clinical, epidemiologic or investigational purposes [2]. In recent years some programmes which allow for the standardisation and indexing of the parameters which must be recorded and used in the bronchoscopic report have been proposed. The Endotorace System [3] and GestEndo Thorax, developed in the 90 s, are among the most popular.

Many modern systems have also integrated the acquisition, filing and handling of pictures and videos which constitute a vital supplementation to the report. Two examples are the 'AreaPneumo' system and the more recent 'EndoPneumo' system which also include the option of using a predefined glossary based on literature [4-6] which has been approved by the Bronchoscopic Working Groups of Italian Scientific Societies [7]. Conversely, the Archivex [8] and Endoics [9] systems for the acquisition and handling of images need to be integrated with a package for the management of electronic clinical files. This package also makes it possible to publish reports on Clinical Data Warehouse which can then be consulted on the corporate intranet, e.g. Infoclin-Eurosoft [10]. However, there are also other centres which have personally developed and utilised diverse systems which are, in general, of optimal quality.

Like all projects which deal with electronic clinical files, the success of a project for an electronic report depends on a long process of reality oriented techniques and a participative approach [11].

The advantages of the compilation of data and images and computerised reports are indisputable:

1. Large amounts of data in a reduced physical space.

2. Simplified standardisation of information through the utilisation of some mandatory fields.

3. Simplified report duplication. (e.g: no need for a separate letter to the referring physician). 
4. Simplified interaction with the system (multimedia, voice recognition, etc.).

5. Remote and simultaneous access possible.

6. Instantaneous availability of data and the possibility of sharing information and images with other specialists for consultation or audit.

7. Enhanced data protection.

8. Utilisation of a code for the retrieval and subsequent analysis of the information (data mining) using a programme for database interrogation.

9. Possibility of transferring and sharing data possible. (Telemedicine)

10. Possibility of user-interactive integration (clinical decision support systems) with signaling of warning and memo.

11. Utilisation of data and images for training purposes.

12. Utilisation of coding for statistical analysis.

Software with the following functions is therefore recommended:

- To interface with the hospital electronic records database (e.g., RIS-PACS).

- To comply with the standard format DICOM for the depiction of the radiological and bronchoscopic images.

- To integrate with a Pneumologic-clinic file for data recording.

- To freely and rapidly interrogate the database using the specific descriptions previously recorded.

- To create an archive of previously formatted reports.

- Completely modify the log.

- Interface for epidemiologic research.

- To use forms for requesting exams which are compatible with your PC.

- To obtain completely personalised reports.

- To immediate export selected data to other statistical platforms.

- To analsise pneumologic activity by the administration.

- To report with high-definition photos.

- To handle images and videos in graphic format compatible with windows, video-endoscopy, VCR, scanner; with direct acquisition of photos and videos in a file with remote access.

- To process both the images captured during the examination and the graphic illustration of the bronchial tree.

- Back-up system.

\section{Recommendations for the preparation} of the computerized bronchoscopic report

The report must include a series of codified mandatory fields, possibly multiple choice questions which allow for an appropriate characteriza- tion and preservation of the data, facilitating statistic and epidemiologic analysis.

\section{A. Procedure and patient information:}

1. Date and place of the test.

2. Patient information.

3. Data of the physicians involved.

4. Referring physician.

\section{B. Medical history:}

1. Chief complaint, present and past medical and surgical history.

2. Exposure to tobacco and industrial fumes. (Social history: e.g.: drugs).

3. Comorbid conditions and risk factors for the bronchoscopic procedure.
a) Cardiovascular.
b) Pulmonary.
c) Diabetes.
d) Mental status alterations.
e) Coagulopathy.

4. Description of relevant findings of imaging diagnostic procedures (CXR, CT, PET), if possible including images.

5. Description of any other diagnostic tests required for the appropriate performance of the procedure, including hemogram, basic metabolic panel, coagulation panel, electrocardiogram and spirometry, etc.).

6. Previous pertinent interventions such as chemotherapy, surgery and/or radiotherapy.

7. Palliative therapy.

\section{Bronchoscopic procedure information:}

1. Date and time of the procedure.

2. Name of the bronchoscopist and/or supervisor.

3 . Priority of the request (urgent versus scheduled).

4. Indications of the test (see appendix 1).

5. Type of procedure (bronchoscopy for diagnosis or treatment, autofluorescence, EBUS, thoracoscopy, type of laser or other instrument utilized for debulking, etc.

6. Instrument utilised.

7. Site of instrument insertion.

8. Any fluoroscopic or computer tomography assistance.

9. Premedication, local or general anesthesia.

a) Drug name.

b) Formulation and route of administration.

c) Total dose.

d) Any anesthesiological assistance. Name of the anesthesiologist.

10. Specifications of the procedure.

- Type (bronchial lavage, bronchial or tracheal biopsy, transbronchial or transtra- 
cheal biopsy, TBNA transbronchial or transtracheal, BAL, brushing, pulmonary biopsy, etc.).

- Site.

- Number of samples.

- Type of analysis of the sample (cytological, hystological, microbiologic, immunologic, etc.).

11. Indications for a subsequent procedure.

\section{Results and conclusions:}

1. Description and localisation of the bronchoscopic findings in the case of a diagnostic bronchoscopy. (Possibly using a code/glossary for each site and pathological characteristics. See Appendix 2a, with the option of adding free text).

2. Description of subsequent treatment, including the type and location of the intervention performed. (Possibly using a code/glossary. See Appendix 2a, with the option of adding free text with the description of the prosthesis utilized and outcome of the intervention).

3. Complications.

4. Brief conclusions

5. Follow up suggestions.

6. Coding: Code of diseasesand procedure either diagnostic or therapeutic for the automatic calculation of the DRG.

\section{F. Bronchoscopic imaging:}

1. Representation of the lesion site or of treatment over a predefined anatomical scheme.

2. Descriptive photography of the lesion.

3. Descriptive video of the test.

\section{G. Description of the sample sent for cyto-hysto- logic and bacteriological examination}

\section{APPENDIX 1: BRONCHOSCOPY INDICATIONS}

\section{A. Diagnostic bronchoscopy:}

- Chronic cough.

- Dyspnea and stridor.

- Hemoptysis.

- Suspicion of pulmonary tumour (see below, abnormal chest-X-ray).

- Abnormal chest-X-ray.

- Mass (solid or cavitated).

- Recurrent pulmonary infiltrate.

- Unresolved pulmonary infiltrate.

- Persistent atelectasis (segmental, lobar or pulmonary).

- Unilateral hyperinflation or radiographic lucency (tumour or foreign body).

- Abnormality of the mediastinum and hilum.
- Pleural effusion.

- Lymphoadenopathy and/or peritracheal mass.

- Diffuse lung infiltrates.

- Persistent pneumothorax.

- Pulmonary infection.

- Diaphragmatic paralysis.

- Vocal cord paralysis and hoarseness.

- Foreign body suspicion.

- Chemical or thermal burn of the tracheo-bronchial tree.

- Refractory pulmonary abscess.

- Thoracic trauma.

- Tracheo-bronchial fistula.

- Atypical cells in the sputum.

\section{B. Therapeutic bronchoscopy:}

- Secretions, mucus plug or retained clot.

- Necrotic tracheo-bronchial mucosa.

- Tracheo-bronchial foreign body.

- Massive hemoptysis.

- Malignant endoluminal tumour.

- Benign endoluminal tumour.

- Inflammatory stenosis of the upper or lower airway.

- Broncho-pleuritic fistula.

- Tracheo or bronco-oesophageal fistula.

- Pulmonary abscess.

- Bronchogenic cyst.

APPENDIX 2: GLOSSARY OF TRACHEOBRONCHIAL LESIONS AND PROCEDURES

\section{Appendix 2a: Tracheobronchial lesions}

\section{Tracheo-bronchial wall}

Normal mucosa:

- Normal trophic properties.

- Normal surface brightness.

Abnormal mucosa because of:

- Paleness.

- Opacity.

- Loss of the normal brightness.

- Hypervascularization.

- Hypovascularization.

- Edema.

- Hyperemia.

- Spontaneous bleeding.

- Petechiae.

- Ulcerations.

- Necrosis.

- Enlargement of the glandular ostium.

- Anthracosis.

- Atrophy.

- Vanishing of mucosal folds.

- Evidence of mucosal folds.

- Cartilage protrusion. 
- Thickening.

- Irregularity.

- Infiltration with:

- irregularity;

- neovascularization;

- necrosis;

- paleness;

- edema;

- hyperemia;

- vanishing of the cartilaginous frame.

- Vegetations:

- nodular;

- multinodular;

- smooth surface;

- irregular surface;

- granular surface;

- polypoid;

- wide implantation base;

- necrotic;

- hypervascularized;

- ulcerated;

- bleeding.

\section{Lumen}

- Stenosis:

- vegetation;

- infiltration;

- extrinsic compression;

- $\quad$ stenosis less than $25 \%$;

- $\quad$ stenosis less than 50\%;

- $\quad$ stenosis more than $50 \%$;

- subtotal stenosis;

- total stenosis;

- concentric stenosis;

- benign, membranous stenosis;

- granulomatous;

- iatrogenic;

- traumatic;

- congenital.

- Obstruction:

- Vegetation.

- Infiltration.

- Extrinsic compression.

- Obstruction less than $25 \%$.

- Obstruction less than $50 \%$.

- Obstruction more than 50\%.

- Subtotal obstruction.

- Total obstruction.

- Concentric obstruction.

- Oval deformation:

- Extrinsic compression.

- Edema.

- Enlargement secondary to:

- atrophy;

- bronchiectasis;

- distension.

- Anomaly of the bronchial Carrefour:
- Enlargement secondary to edema.

- Enlargement secondary to extrinsic compression.

- Enlargement secondary to infiltration.

- Enlargement secondary to distension.

- Enlargement secondary to scaring.

- Roundness secondary to edema.

- Roundness secondary to extrinsic compression.

- Roundness secondary to infiltration.

- Roundness secondary to distension.

- Roundness secondary to scaring.

- Thinning secondary to atrophia.

- Surgical site:

- Surgical site with normal suture.

- Surgical site with granulomatous suture.

- Surgical site with infiltrated suture.

- Chemotherapy site.

- External radiotherapy site.

- Endoluminal radiotherapy site.

- Tuberculoid scar site.

- Scarred site after inhalation of toxic substances.

- Fistula:

- Congenital tracheo-oesophageal fistula: $\checkmark$ Traumatic tracheo-oesophageal fistula.

$\checkmark$ Neoplastic tracheo-oesophageal fistula.

- Congenital broncho-oesophageal fistula.

- Traumatic broncho-oesophageal fistula.

- Neoplastic broncho-oesophageal fistula.

- Traumatic broncho-pleural fistula.

- Surgically related broncho-fistula.

- Tracheo-bronchomalacia.

- Abnormal substance:

- Absent.

- Abnormal secretions.

- Bleeding.

- Calculi.

- Foreign bodies.

- Disorder of the bronchial movement:

- Abnormal movements during respiration.

- Abnormal movement during cough.

\section{Appendix 2b: Tracheobronchial procedures}

\section{A. Diagnostic}

1. Aspiration (A).

2. Transbronchial needle aspiration (TBNA).

3. Transtracheal needle aspiration (TTNA).

4. Broncho-alveolar lavage (BAL).

5. Bronchial biopsy (BB).

6. Transbronchial pulmonary biopsy (TBB).

7. Tracheal biopsy (TB).

8. Transbronchial needle biopsy (TBNB).

9. Transtracheal needle biopsy (TTNB).

10. Brushing for cytology (BrC).

11. Brushing sterile (BrC). 


\section{B. Therapeutic}

1. Laser assisted debulking.

2. Stent implantation.
a) Metallic.
b) Siliconic.
c) Hibrid.

3. Tracheobronchial dilatation.

4. Endoluminal prosthesis reposition.

5. Brachytherapy.

6. Photodynamic therapy.

7. Electrocauterisations.

8. Cryotherapy.

9. Endoscopic lung volume reduction.

i. Region of origin.

ii. City of origin.

\section{References}

1. Trosini-Désert V, Morin JM, Fournier C, Similowski T, Vergnon JM. Le Groupe d'endoscopie bronchique de langue française. The flexible fiberoptic bronchoscopy report: medico-legal issues. Rev Mal Respir 2010; 7: 729-40.

2. British Thoracic Society Bronchoscopy Guidelines Committee, a Subcommittee of Standards of Care Committee of British Thoracic Society. British Thoracic Society guidelines on diagnostic flexible bronchoscopy. Thorax 2001; 56 Suppl 1: i1-21.

3. Trevisani L, Sartori S, Putinati S, Milani G, Fiorillo E. Data processing in endoscopy: Endotorace, a new software program for bronchoscopy reporting. Endoscopy 1994; 26: 631.

4. Ikeda S. Atlas of flexible bronchofiberscopy. George Thieme Publishers, Stuttgart, 1974.

5. Valenti S, Scordamaglia A, Crimi P, Mereu C. La fibrobroncoscopia e le sue applicazioni cliniche. Ed. Minerva Medica, Torino, 1980.

6. Prakash UBS. Bronchoscopy. Raven Press, Mayo Foundation, New York, 1994.

7. www.endopneumo.it

8. www.archivex.it

9. www.ics-srl.com

10. www.dedalus.eu

11. http://progetti.pneumologia-interventistica.it

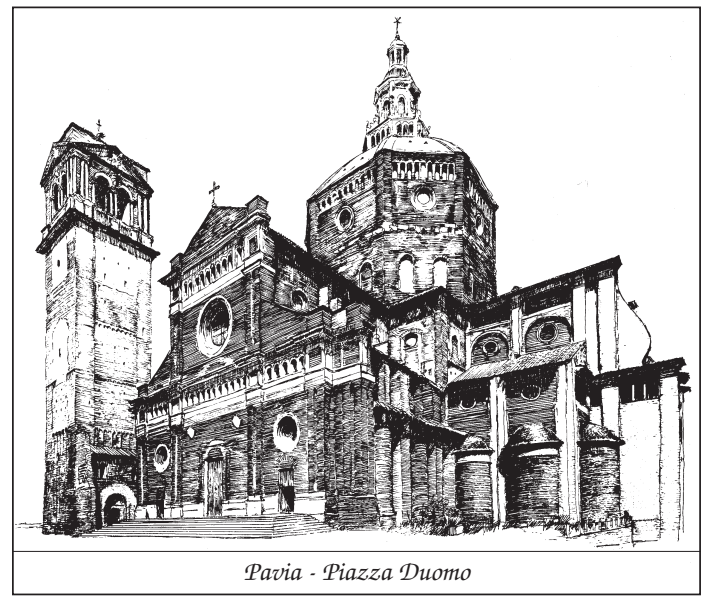

